\title{
Sensor Position Errors Estimating for Moving Sensors by Using Calibration Emitters
}

\author{
Kaiqiang Ren* and Zhengbo Sun \\ National Key Laboratory of Science and Technology on Blind Signal Processing, Chengdu, Sichuan, 86-610041, China \\ ${ }^{*}$ Corresponding author
}

\begin{abstract}
This paper proposed a novel calibration algorithm for sensor position errors of moving sensors by using calibration emitters. The algorithm presented is based on time difference of arrival (TDOA) measurements received by sensor network with sensor position error. Firstly, the linear relation between sensor position errors and TDOA measurements is derived by Taylor's series expansion. Then, a linear minimum mean square error (LMMSE) estimator of sensor position errors is derived based on the linear model. At last, on the assumption that sensor position errors of moving sensors are steady in a short time, a sequential LMMSE algorithm for sensor position errors estimation is proposed. Simulation results demonstrate the good performance of the proposed algorithm.
\end{abstract}

Keywords-sensor position errors; moving sensors; time difference of arrival (TDOA); calibration emitters; sequential linear minimun mean square error (LMMSE)

\section{INTRODUCTION}

Source localization of a signal source is a fundamental problem in many signal processing applications, such as radar, navigation, search and rescue [1-3]. A large amount of localization algorithms using TDOAs have been proposed [4-9] Most of these methods are able to achieve the Cramer-Rao lower bound (CRLB) accuracy on the assumption that the sensor positions are known exactly. Unfortunately, in most cases of practical applications, sensor position errors are unavoidable. The localization accuracy of these methods would be degraded significantly and no longer reach the CRLB accuracy.

Recently, in order to reduce and eliminate the influence of sensor position errors on source localization, a number of calibration algorithms have been proposed [10-14]. On the assumption that the sensor position errors have a statistics of zero-mean Gaussian characteristic, [10] has developed an improved solution. Although this method can reach CRLB accuracy, the localization accuracy is much worse than the one without sensor position errors. [11] studies the use of a single calibration emitter whose position is known exactly to reduce the loss in localization accuracy due to sensor position errors, this method is shown to reach the CRLB accuracy when the source is distant from the sensor array or when the signal-tonoise ratio (SNR) is sufficiently high. It is proved that a single calibration emitter is not able to completely eliminate the influence of sensor position errors on source localization accuracy. [12] extends the work in [11] by investigating the use of multiple calibration emitters to improve the source localization accuracy further. [13-16] analyze the performance of differential calibration algorithm by using calibration emitters. It is shown that differential calibration algorithm can completely remove system errors, but is not able to calibrate sensor position error efficiently.

In this paper, we proposed a novel calibration algorithm for sensor position errors of moving sensor by using calibration emitters. On the assumption that sensor position errors of moving sensors are steady in a short time, we exploit the sequential LMMSE algorithm to estimate sensor position errors. Simulation results demonstrate that the proposed sequential algorithm is more accurate and need less calibration emitters in comparison with the non-sequential algorithm.

\section{PROBLEM Formulation}

Assume there are $M$ moving sensors and $N$ calibration emitters in the localization scenario. The positions of the calibration emitters are denoted as $\mathbf{c}_{j}, j=1,2, \cdots, N$ which are known exactly. The true sensor positions $\mathbf{s}_{i}^{o}$ are not known and only the inaccurate sensor positions $\mathbf{s}_{i}$ are available. Mathematically, we have

$$
\mathbf{s}_{i}=\mathbf{s}_{i}^{o}+\boldsymbol{\delta}_{i}, i=1,2, \cdots, M
$$

where $\boldsymbol{\delta}_{i}$ is the position error in $\mathbf{s}_{i}$. The collection of $\mathbf{s}_{i}$ forms the $3 M \times 1$ sensor position vector

$$
\mathbf{s}=\mathbf{s}^{o}+\boldsymbol{\delta}
$$

where $\mathbf{s}=\left[\mathbf{s}_{1}^{T}, \mathbf{s}_{2}^{T}, \ldots, \mathbf{s}_{M}^{T}\right]^{T}, \quad \mathbf{s}=\left[\mathbf{s}_{1}^{o T}, \mathbf{s}_{2}^{o T}, \ldots, \mathbf{s}_{M}^{o T}\right]^{T}$. We shall assume that $\boldsymbol{\delta}=\left[\boldsymbol{\delta}_{1}^{T}, \boldsymbol{\delta}_{2}^{T}, \ldots, \boldsymbol{\delta}_{M}^{T}\right]^{T}$ is a zero-mean Gaussian vector with covariance matrix $\mathbf{Q}_{s}$.

The TDOAs of the received signals with respect to a reference sensor are estimated. Without loss of generality, we set the first sensor as the reference sensor. After multiplying by the known signal propagation speed $c$, the range difference of arrivals (RDOA) measurements of the signal emitted from the $j$ th calibration emitter between the $i$ th sensor and the 1 th sensor can be denoted as 


$$
m_{i 1}^{j}=r_{i 1}^{j}+n_{i 1}^{j}
$$

where $m_{i 1}^{j}$ is the RDOA measurements with noise $n_{i 1}^{j} \cdot r_{i 1}^{j}$ is the true RDOA, and

$$
r_{i 1}^{j}=\left\|\mathbf{c}_{j}-\mathbf{s}_{i}^{o}\right\|-\left\|\mathbf{c}_{j}-\mathbf{s}_{1}^{o}\right\|
$$

For notation simplicity, we collect $m_{i 1}^{j}, i=1,2, \cdots, M$, $j=1,2, \cdots, N$ to form the $(M-1) N \times 1$ RDOA vector as

$$
\mathbf{m}=\mathbf{r}+\mathbf{n}
$$

where

$$
\begin{gathered}
\mathbf{m}=\left[m_{21}^{1}, \cdots, m_{M 1}^{1}, m_{21}^{2}, \cdots m_{M 1}^{N-1}, m_{21}^{N}, \cdots, m_{M 1}^{N}\right]^{T} \\
\mathbf{r}=\left[r_{21}^{1}, \cdots, r_{M 1}^{1}, r_{21}^{2}, \cdots r_{M 1}^{N-1}, r_{21}^{N}, \cdots, r_{M 1}^{N}\right]^{T} \\
\mathbf{n}=\left[n_{21}^{1}, \cdots, n_{M 1}^{1}, n_{21}^{2}, \cdots n_{M 1}^{N-1}, n_{21}^{N}, \cdots, n_{M 1}^{N}\right]^{T} .
\end{gathered}
$$

$\mathbf{n}$ is assumed to be a zero-mean Gaussian vector with covariance matrix $\mathbf{Q}_{m}$.

\section{SENSOR POSITION ERRoRs Estimate Algorithm}

It is obvious that relation between the unknown sensor position errors and the known measurements is nonlinear. In order to simplify the problem, we should make it a linear relation. Since $\mathbf{s}_{i}^{o}$ are not known and only $\mathbf{s}_{i}$ are available, we substitute (1) into (4) and apply Taylor-series expansion up to first-order term to derive

$$
r_{i 1}^{j} \approx\left\|\mathbf{c}_{j}-\mathbf{s}_{i}\right\|-\left\|\mathbf{c}_{j}-\mathbf{s}_{1}\right\|+\boldsymbol{\rho}_{\mathbf{c}_{j}, \mathbf{s}_{i}}^{T} \boldsymbol{\delta}_{i}-\boldsymbol{\rho}_{\mathbf{c}_{j}, \mathbf{s}_{1}}^{T} \boldsymbol{\delta}_{1}
$$

where $\boldsymbol{\rho}_{\mathbf{c}_{j}, \mathbf{s}_{i}}$ is the unit vector from $\mathbf{s}_{i}$ to $\mathbf{c}_{j}$ and can be denoted as

$$
\boldsymbol{\rho}_{\mathbf{c}_{j}, \mathbf{s}_{i}}=\frac{\left\|\mathbf{c}_{j}-\mathbf{s}_{i}\right\|}{\mathbf{c}_{j}-\mathbf{s}_{i}}
$$

Substituting (6) into (3), we arrive at

$$
m_{i 1}^{j}-\left\|\mathbf{c}_{j}-\mathbf{s}_{i}\right\|-\left\|\mathbf{c}_{j}-\mathbf{s}_{1}\right\|=\boldsymbol{\rho}_{\mathbf{c}_{j}, \mathbf{s}_{i}}^{T} \boldsymbol{\delta}_{i}-\boldsymbol{\rho}_{\mathbf{c}_{j}, \mathbf{s}_{1}}^{T} \boldsymbol{\delta}_{1}+n_{i 1}^{j}
$$

Expressing (8) in matrix form, we can obtain

$$
\mathbf{h}=\mathbf{G} \boldsymbol{\delta}+\mathbf{n}
$$

where

$$
\mathbf{h}_{j}=\left[m_{21}^{j}-\left\|\mathbf{c}_{j}-\mathbf{s}_{2}\right\|-\left\|\mathbf{c}_{j}-\mathbf{s}_{1}\right\|, \cdots, m_{M 1}^{j}-\left\|\mathbf{c}_{j}-\mathbf{s}_{M}\right\|-\left\|\mathbf{c}_{j}-\mathbf{s}_{1}\right\|\right]^{T}
$$

$$
\mathbf{h}=\left[\mathbf{h}_{1}^{T}, \mathbf{h}_{2}^{T}, \cdots \mathbf{h}_{N}^{T}\right]^{T}
$$

$$
\mathbf{G}_{j}=\left[\begin{array}{ccccc}
-\boldsymbol{\rho}_{\mathbf{c}_{j}, \mathbf{s}_{1}} & -\boldsymbol{\rho}_{\mathbf{c}_{j}, \mathbf{s}_{2}} & & & \\
-\boldsymbol{\rho}_{\mathbf{c}_{j}, \mathbf{s}_{1}} & & -\boldsymbol{\rho}_{\mathbf{c}_{j}, \mathbf{s}_{3}} & & \\
\vdots & & & \ddots & \\
-\boldsymbol{\rho}_{\mathbf{c}_{j}, \mathbf{s}_{1}} & & & & -\boldsymbol{\rho}_{\mathbf{c}_{j}, \mathbf{s}_{M}}
\end{array}\right]
$$

$$
\mathbf{G}=\left[\mathbf{G}_{1}^{T}, \mathbf{G}_{2}^{T}, \cdots \mathbf{G}_{N}^{T}\right]^{T}
$$

Based on the Bayesian Gauss-Markov theorem, the LMMSE estimator of $\boldsymbol{\delta}$, denoting it as $\widehat{\boldsymbol{\delta}}$, is

$$
\widehat{\boldsymbol{\delta}}=\left(\mathbf{Q}_{s}^{-1}+\mathbf{G}^{T} \mathbf{Q}_{m}^{-1} \mathbf{G}\right)^{-1} \mathbf{G}^{T} \mathbf{Q}_{m}^{-1} \mathbf{h}
$$

Subtracting the estimated sensor position errors in (10) from $\mathbf{s}$, we can obtain the improved sensor position as

$$
\widehat{\mathbf{S}}=\mathbf{s}-\widehat{\boldsymbol{\delta}}_{\mathbf{s}}=\mathbf{s}^{o}+\boldsymbol{\delta}_{\mathbf{s}}-\widehat{\boldsymbol{\delta}}_{\mathbf{s}}
$$

Since $\widehat{\boldsymbol{\delta}}$ is a LMMSE estimator, the covariance matrix of $\widehat{\mathbf{s}}$ is

$$
\operatorname{cov}(\widehat{\mathbf{s}})=\operatorname{cov}\left(\widehat{\boldsymbol{\delta}}_{\mathbf{s}}-\boldsymbol{\delta}_{\mathbf{s}}\right)=\left(\mathbf{Q}_{s}^{-1}+\mathbf{G}^{T} \mathbf{Q}_{m}^{-1} \mathbf{G}\right)^{-1}
$$

In order to analysis the error performance of the improved sensor position $\widehat{\mathbf{s}}$, we have

$$
\begin{aligned}
\operatorname{cov}(\mathbf{s})-\operatorname{cov}(\widehat{\mathbf{s}}) & =\mathbf{Q}_{s}-\left(\mathbf{Q}_{s}^{-1}+\mathbf{G}^{T} \mathbf{Q}_{m}^{-1} \mathbf{G}\right)^{-1} \\
& =\mathbf{Q}_{s} \mathbf{G}^{T}\left(\mathbf{Q}_{m}^{-1}+\mathbf{G} \mathbf{Q}_{s} \mathbf{G}^{T}\right)^{-1} \mathbf{G} \mathbf{Q}_{s}
\end{aligned}
$$

It is obvious that (13) is a positive semidefinite matrix. Thus we have the conclusion that the improved sensor position is at least as good as, if not better than, the original one.

\section{SEqUential LMMSE Algorithm}

The LMMSE algorithm proposed in section III can reach high accuracy on the condition that the number of calibration emitters is large enough. Unfortunately, the number of calibration emitters is severely limited in practical applications, especially in geolocation. On the assumption that sensor position errors of moving sensors are steady in a short time, we exploit the sequential LMMSE algorithm to estimate sensor 
position errors by using the TDOA measurements in different time. Since the sensors are moving, the TDOA measurements in different time are corresponding to different geometry, which is equivalent to calibration emitters in different positions

The proposed sequential LMMSE algorithm for sensor position errors is as follow

i. Initialize the estimate of sensor position errors and the covariance of it.

$$
\begin{gathered}
\widehat{\boldsymbol{\delta}}^{(0)}=E(\boldsymbol{\delta})=0 \\
\mathbf{Q}^{(0)}=E\left[\left(\boldsymbol{\delta}-\widehat{\boldsymbol{\delta}}^{(0)}\right)\left(\boldsymbol{\delta}-\widehat{\boldsymbol{\delta}}^{(0)}\right)^{T}\right]=\mathbf{Q}_{s}
\end{gathered}
$$$$
k=0
$$

ii. While $\Delta \mathbf{Q}^{(k)}=\operatorname{trace}\left(\mathbf{Q}^{(k)}-\mathbf{Q}^{(k-1)}\right)>\boldsymbol{\varepsilon}$ do

$$
\begin{gathered}
k=k+1 \\
\mathbf{B}^{(k)}=\mathbf{Q}^{(k-1)} \mathbf{G}^{(k)}\left(\mathbf{Q}_{m}+\mathbf{G}^{(k)} \mathbf{Q}^{(k-1)} \mathbf{G}^{(k) T}\right) \\
\widehat{\boldsymbol{\delta}}^{(k)}=\widehat{\boldsymbol{\delta}}^{(k-1)}+\mathbf{B}^{(k)}\left[\mathbf{h}^{(k)}-\mathbf{G}^{(k)} \widehat{\boldsymbol{\delta}}^{(k-1)}\right] \\
\mathbf{Q}^{(k)}=\left(\mathbf{I}-\mathbf{B}^{(k)} \mathbf{G}^{(k)}\right) \mathbf{Q}^{(k-1)}
\end{gathered}
$$

end

$$
\text { iii. } \quad \widehat{\boldsymbol{\delta}}=\widehat{\boldsymbol{\delta}}^{(k)}
$$

In the algorithm proposed above, $\varepsilon$ is iteration threshold, $\mathbf{G}^{(k)}$ and $\mathbf{h}^{(k)}$ is defined as in (9) according to the $k$ th TDOA measurements and the corresponding sensor position. $\mathbf{I}$ is an identity matrix in property size.

\section{SimUlations}

In this section, we shall verify the performance of the proposed sequential LMMSE algorithm through simulation. The simulation scenario includes 3 sensors and 3 calibration emitters. The true sensor positions are listed in Table 1 and the known positions of calibration emitters are listed in Table 2. For simplification, we assume that only the 1th sensor is moving with velocity $\mathbf{v}=[100,100]^{T} \mathrm{~m} / \mathrm{s}$ and the others are stationary. The RDOA measurements are created by adding to the true values zero-mean white Gaussian noise with covariance matrix $\mathbf{Q}_{m}=\sigma_{m}^{2} \mathbf{I}$, where $\sigma_{m}=1 e-9{ }^{*} c \mathrm{~m}$ and $\mathbf{I}$ is an identity matrix. We assume that sensor position errors are steady in an iteration process, but the noisy sensor positions in different iteration process are created by adding to the true values zero-mean white Gaussian noise with covariance matrix $\mathbf{Q}_{s}=\sigma_{s}^{2} \mathbf{I}$, where $\sigma_{s}=300 \mathrm{~m}$.

TABLE I. TRUE POSITIONSOF SENSORS (IN KILOMETERS)

\begin{tabular}{cc}
\hline sensor no. $i$ & $\mathbf{s}_{i}^{o}$ \\
\hline 1 & $(0,0)$ \\
2 & $(10,0)$ \\
3 & $(0,10)$ \\
\hline
\end{tabular}

TABLE II. CALIBRATION EMITTER POSITIONS (IN KILOMETERS)

\begin{tabular}{cc}
\hline $\begin{array}{c}\text { calibration emitters } \\
\text { no. } j\end{array}$ & $\mathbf{c}_{j}$ \\
\hline 1 & $(50,80)$ \\
2 & $(-100,10)$ \\
3 & $(-60,30)$ \\
\hline
\end{tabular}

Figure 1 plots sensor position errors estimating accuracy in iteration process with different amount of calibration emitters. We use the Euclidean-distance between real sensor position errors and the estimating sensor position errors to assess the estimating accuracy, which is defined as $D\left(\boldsymbol{\delta}, \widehat{\boldsymbol{\delta}}^{(k)}\right)=\left\|\boldsymbol{\delta}-\widehat{\boldsymbol{\delta}}^{(k)}\right\|$. When there is only the 1th calibration emitter, the iteration result may converge to locally optimal solution. When the amount of calibration emitters is more than one, the proposed sequential LMMSE algorithm doesn't suffer from local convergence problem. By contrasting the case of 2 calibration emitters with the case of 3 calibration emitters, it can be shown that estimating accuracy is not affected with the amount of calibration emitters. On the contrary, convergence rate of the proposed algorithm is severely affected with the amount of calibration emitters, the bigger the amount of calibration emitters is, the faster the convergence rate is.
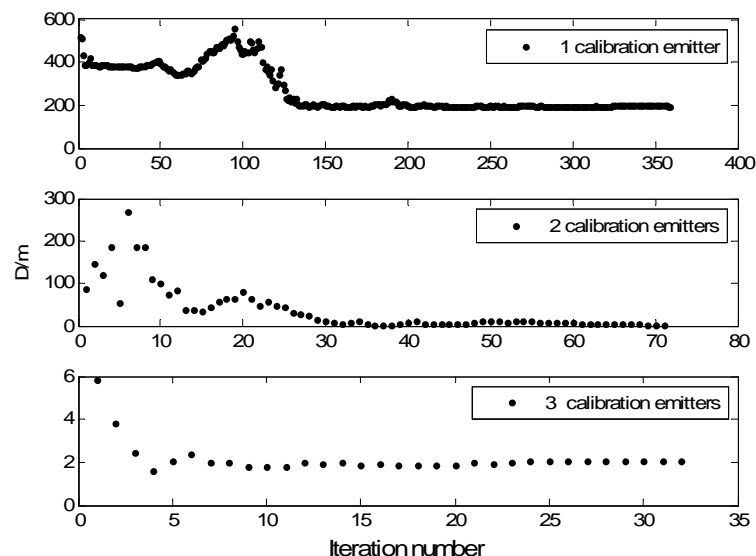

FIGURE I. ESTIMATING ACCURACY IN ITERATION PROCESS

To illustrate the good performance of the proposed sequential LMMSE algorithm, a comparison between the estimating accuracy of sequential LMMSE algorithm and that of LMMSE algorithm is made when there are 2 calibration emitters. Figure 2 shows that the estimating accuracy of LMMSE algorithm is almost invariable with the changing of TDOA measurements. On the contrary, the estimating accuracy of sequential LMMSE algorithm is becoming more accuracy 
with the iteration number increasing. It is obvious the estimating accuracy of sequential LMMSE algorithm is more accuracy than that of LMMSE algorithm.

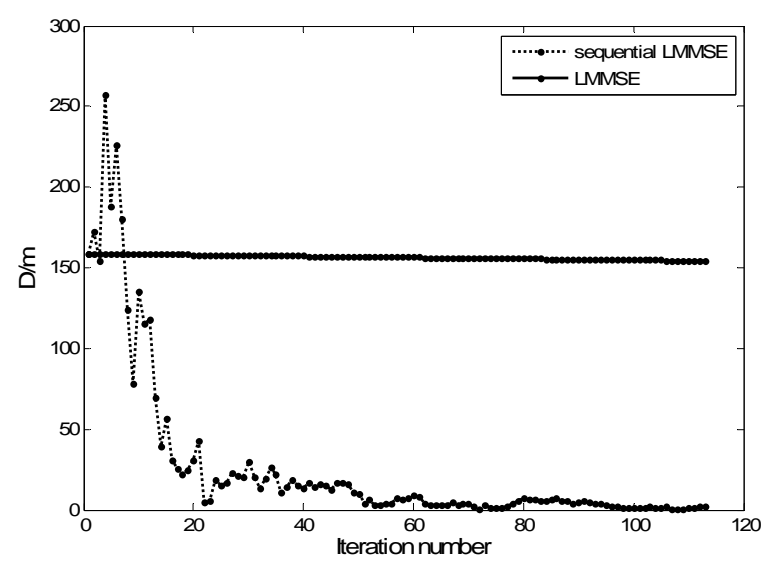

FIGURE II. COMPARISON BETWEEN SEQUENTIAL LMMSE AND LMMSE

\section{CONCLUSION}

In this paper, we proposed a novel calibration algorithm for sensor position errors of moving sensors by using calibration emitters. At first, we derive the linear relation between sensor position errors and TDOA measurements by Taylor's series expansion. Then we establish a LMMSE estimator for sensor position errors based on the linear model. At last, on the assumption that sensor position errors of moving sensors are steady in a short time, the sequential LMMSE algorithm is exploited to estimate sensor position errors. Simulation results shows that the proposed sequential LMMSE algorithm for sensor position errors is more accuracy and need less amount of calibration emitters than LMMSE algorithm.

\section{REFERENCES}

[1] T. T. Ha and R. C. Robertson. "Geostationary satellite navigation systems,” IEEE Trans on Aerosp Electron Syst, vol., no.23, pp.247-254, 1987.

[2] T. Li, A. Ekpetryong, Y. F. Huang. "Source localization and tracking using distributed asynchronous sensors," IEEE Trans on Signal Process, vol.54, no.10, pp.3991-4003, 2006.

[3] K. C. Ho and M. Sun. "An accurate algebraic closed-form solution for energy-based source localization,” IEEE Trans on Audio Speech Lang Process, vol.15, pp.2542-2550.2007.

[4] D. Carevic. "Automatic estimation of multiple target positions and velocities using passive TDOA measurements of transients," IEEE Trans on Signal Processing, vol.55, no.2, pp.424-436, 2007.

[5] L. Wang, T. K. Hon, J. D. Reiss and A. Cavallaro. "Self-localization of Ad-Hoc arrays using time difference of arrivals,” IEEE Trans on Signal Processing, vol.64, no.4, pp.1018-1033, 2016.

[6] Y. T. Chan, K. C. Ho. "A simple and efficient estimator for hyperbolic location,” IEEE Trans on Signal Processing, vol.42, pp.1905-1915, 1994.

[7] S Ming and Ho K.C.. "An asymptotically efficient estimator for TDOA and FDOA positioning of multiple disjoint sources in the presence of sensor location uncertainties,” IEEE Trans. Signal Process., vol.59, no.7, pp. 3434-3440, 2011

[8] Z. Guohui, F. Dazheng, X. Hui and Z. Yan. “An approximately efficient bi-iterative method for source position and velocity estimation using
TDOA and FDOA measurements,” Signal Processing, no.125, pp.110121, 2016

[9] B. Yang, J. Scheuing. "Cramer-Rao bound and optimum sensor array for source localization from time difference of arrival," in Proc. IEEE ICASSP, vol.4, pp.961-964, 2005.

[10] K. C. Ho, X. Lu and L. Kovavisaruch, "Source localization using TDOA and FDOA measurements in the presence of recevier location errors: analysis and solution,” IEEE Trans on Signal Processing, vol.55, no.2, pp.684-696, 2007.

[11] K. C. Ho, Le Yang. "On the use of a calibration emitter for source localization in the presence of sensor position uncertainty," IEEE Trans on Signal Processing, vol.56, no.12, pp.5758-5772, 2008.

[12] Le Yang, K. C. Ho. "Alleviating sensor position error in source localization using calibration emitters at inaccurate locations," IEEE Trans on Signal Processing, vol.58, no.1, pp.67-83, 2010.

[13] S. F. Yao, Q. He and J. Y. Xiong. "Correcting accuracy analysis of multi-sensor TDOA location based on reference stations," Computer Science, vol.39, no.11, pp.79-82, 2012.

[14] W. J. Ren, S. Y. Lv and D. F. Shi. "Error analysis of position calibration technique for satellite TDOA location system," Telecommunication Engineering, vol.55, no.5, pp.503-508, 2014.

[15] L. H. Guo, F. C. Guo and J. Z. Li. "A method using multiple calibration for orbit companion satellite TDOA and FDOA geo-location system," Journal of Astronautics, vol.33, no.10, pp.1407-1412, 2012.

L. Jinzhou, G. Fucheng, Y. Le, J. Wenli, and P. Hongwei. "On the use of calibration sensors in source localization using TDOA and FDOA measurements,” Digital Signal Processing, no.27, pp.33-43, 2014. 